\title{
Les champignons sauvages comestibles et connaissances endogènes des peuples autochtones Mbènzèlè et Ngombe de la République du Congo
}

\author{
Sydney T. NDOLO EBIKA ${ }^{1,2^{*}}$, Jean E.I. CODJIA ${ }^{3}$, Nourou S. YOROU ${ }^{3}$ et ATTIBAYEBA ${ }^{1}$ \\ ${ }^{1}$ Faculté des Sciences et Techniques, Université Marien NGOUABI, B.P. 69, Brazzaville, République du Congo ; \\ ${ }_{2}^{2}$ nitiative des Champignons et des Plantes du Congo (ICPC), BP 2300, Brazzaville, République du Congo ; \\ 'Laboratoire d'Écologie, de Botanique et de Biologie Végétale (LEB), Université de Parakou, 03 BP 125, Parakou, Bénin \\ Corresponding author: ndoloicpc@gmail.com
}

Original submitted in on $16^{\text {th }}$ March 2018. Published online at www.m.elewa.org on $30^{\text {th }}$ June 2018 https://dx.doi.org/10.4314/jab.v126i1.5

\section{RÉSUMÉ}

Objectifs : Cette étude vise à sauvegarder les connaissances endogènes sur l'utilisation des champignons de forêts en République du Congo et à documenter scientifiquement ces ressources naturelles encore très peu connues.

Méthodologie et Résultats: Les collectes opportunistes et les enquêtes ethnomycologiques au sein des peuples autochtones Mbènzèlè et Ngombe ont permis de recueillir des informations sur les différents types d'usage des champignons, leurs noms vernaculaires et leur signification. Au total, 25 genres et 51 espèces de champignons comestibles ont été enregistrés dans le milieu d'étude dont 32 ont été citées lors des enquêtes. Les genres les plus représentées sont Cantharellus, Marasmius, Russula et Termitomyces. Trois espèces sont utilisées dans la pharmacopée traditionnelle et sept ont fait l'objet de vente ou d'échange sous forme de Troc.

Les connaissances sur les espèces de champignons sont détenues par un petit groupe au sein des enquêtés de chaque groupe ethnique. Les résultats de cette étude ne représentant qu'environ $50 \%$ de la connaissance endogène de ces deux groupes ethniques étudiés, poussent à intensifier des missions de terrain auprès de ces populations afin de conserver ces connaissances traditionnelles sur les Champignons Sauvages Comestibles (CSC).

Conclusions et application des résultats. L'issue des tels travaux futurs sera la réalisation d'un guide sur les CSC en République du Congo. Un tel ouvrage contribuerait à la meilleure connaissance de la mycoflore africaine.

Mots clés : Bomassa, connaissances traditionnelles, Produits Forestiers Non Ligneux, macromycètes, Makao, Forêts du Nord Congo, Troc. 
Ebika et al, J. Appl. Biosci. 2018 Les champignons sauvages comestibles et connaissances endogènes des peuples autochtones Mbènzèlè et Ngombe de la République du Congo.

Wild Edible Fungi and indigenous knowledge of Mbènzèlè and Ngombe populations in the Republic of Congo

\section{ABSTRACT}

Objectives: This study aims at safeguarding indigenous knowledge on the use of forest fungi and scientifically documenting those natural resources yet not very well known in the Republic of Congo.

Methodology and Results: Opportunistic collecting and ethnomycological interviews in Mbènzèlè and Ngombe populations enabled to obtain information on the uses of fungi, their local names and meaning. In total, 25 genera and 51 species of edible fungi were recorded in the study area of which 32 were cited during interview. The most represented genera are Cantharellus, Marasmius, Russula et Termitomyces. Three species are used in traditional medicine and seven were sold or exchanged as in the form of barter.

Knowledge on fungal species is hold by a small group of people within each ethnical group. Results of this study only representing about $50 \%$ of the indigenous knowledge of the ethnical groups studied lead to intensify fieldworks with those populations in order to conserve traditional knowledge on the Wild Edible Fungi (WEF) in the future.

Conclusions et application of findings: The outcome of such works will be to produce a guide of WEF in the Republic of Congo. Such a document will contribute to a better understanding of the african mycoflora.

Keywords: Bomassa, traditional knowledge, Non Woody Forest Products, macrofungi, Makao, Forests of Northern Congo, Barter.

\section{INTRODUCTION}

En Afrique Centrale, le nombre des Peuples Autochtones (PA) est estimé à environ 919,500 (Olivero et al., 2016) dont la grande partie, soit environ 500,000 (Seshadri, 2005), se trouverait en République Démocratique du Congo suivi du Gabon, de la République du Congo, du Cameroun et de la République Centrafricaine (Olivero et al., 2016). Sept principaux groupes des PA sont connus en Afrique Centrale dont les Aka, les Baka, les Bongo, les Cwa, les Gyeli, les Mbuti et les Twa (Bahuchet \& Thomas, 1986). Les Baka et Bangombe sont rencontrés au Sud-Est du Cameroun, Nord du Gabon et NordOuest de la République du Congo tandis que les BaAka (ou encore Bangombe) et BaMbènzèlè (les Baka) sont rencontrés en République centrafricaine et au Nord du Congo (Bahuchet, 1991). Dans cette partie du Congo, deux groupes démographiques sont reconnus: les autochtones (Bangombe et

\section{MATÉRIEL ET MÉTHODE}

Milieu d'étude : L'étude a été menée au sein des villages Bomassa dans le Départements de la Sangha, Makao et Thanry-Congo (distants de $5 \mathrm{~km}$ ) dans le Département de la Likouala (Fig. 1).

Végétation: Conformément à la subdivision phytogéographique du Congo (Kimpouni et al., 1992), notre zone d'étude fait partie du district
BaMbènzèlè) qui sont des chasseurs-cueilleurs et les bantous qui sont des agriculteurs (Bouka Owoko \& N'zobo, 2011). Ces deux groupes démographiques entretiennent des relations basées sur les échanges, du travail de champs et des activités de chasse commune (Bahuchet \& Thomas, 1986). Les PA Mbènzèlè et Ngombe tirent de la forêt la majeure partie de leur nourriture telle que la viande, les chenilles, les fruits et les champignons (Bahuchet, 1991; Lewis, 2002). Les champignons sauvages comestibles (CSC) étant encore très mal étudiés au Congo (Boa, 2006), nous nous sommes intéressés à étudier ces CSC auprès des Mbènzèlè et des Ngombe afin de (i) connaître la diversité des champignons consommés dans cette partie d'Afrique Centrale et de (ii) sauvegarder les connaissances traditionnelles des Mbènzèlè et des Ngombe face aux différents changements de culture et d'habitat.

phytogéographique de la Haute Sangha et se caractérise par trois sous-types de végétation à savoir les forêts mixtes de terre ferme (FMTF), les marécages et les forêts monodominantes à Gilbertiodendron dewevrei (De Wild.) J. Léonard (Moutsamboté et al., 1994). Les FMTF sont des formations hétérogènes caractérisées par des espèces d'arbres de grande taille telles que Celtis 

des peuples autochtones Mbènzèlè et Ngombe de la République du Congo.

mildbraedii Engl., Entandrophragma spp. (Harris, 2002) tandis que les forêts monodominantes à Gilbertiodendron dewevrei (FGD) se rencontrent très souvent le long des cours d'eau (Vennetier, 1966; Moutsamboté et al., 1994; Harris, 2002; Gillet \& Doucet, 2012). Nos récoltes ont essentiellement été faites dans les FMTF et les FGD.

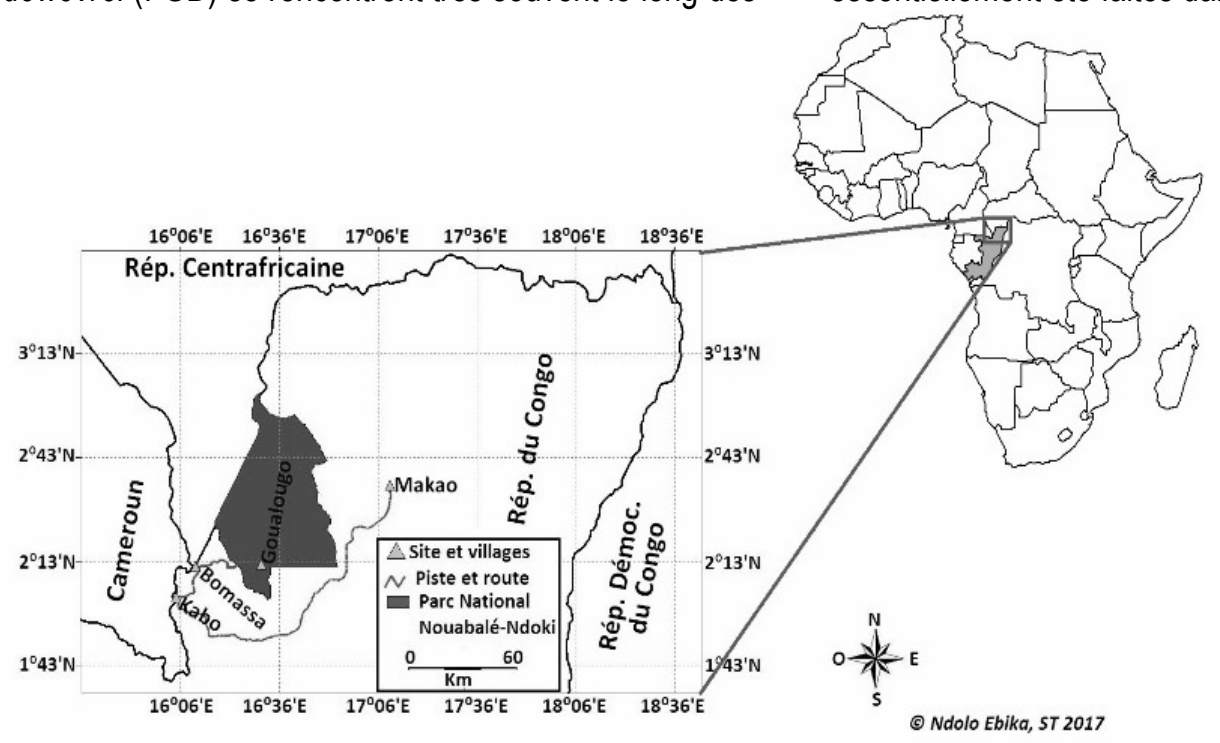

Figure 1 : Localisation de la zone d'étude.

Échantillonnage de la population : L'étude préliminaire sur les Produits Forestiers Non Ligneux (PFNL) menée à Bomassa, Makao et Thanry-Congo (Ndolo Ebika et al., 2013a; 2013b) a montré que les Mbènzèlè et les Ngombe ont une importante connaissance des ressources naturelles qu'ils utilisent quotidiennement et représentent, par conséquent, les principaux fournisseurs et ravitailleurs de ces localités en PFNL tels que les feuilles de Gnetum africanum Welw., G. buchholzianum Engl., les fruits sauvages et les champignons. Ces résultats nous ont orientés vers le choix des Mbènzèlè et les Ngombe comme population à enquêter. Les enquêtes ont été menées sur un échantillon de quarante et quatre (44) composé, pour les femmes et jeunes filles des deux groupes, de 06 jeunes, 13 adultes et 08 vieillards et, pour les hommes, de 1 jeune, 9 adultes et 7 vieillards.

Collecte des données mycologiques: Les récoltes opportunistes en forêt avec des guides Mbènzèlè ou Ngombe couplées avec des enquêtes au niveau des villages ont permis de collecter les spécimens et de recueillir les informations sur leurs utilisations, leurs noms vernaculaires en langue Mbènzèlè ou Ngombe ainsi que la signification de ces noms. Ces spécimens ont été séchés le même jour en utilisant un réchaud à pétrole de 2 litres de contenance et un séchoir spécialement conçu pour le séchage des champignons en milieu tropical (De Kesel, 2001). Une fois secs, les spécimens étaient emballés dans des sachets plastiques de type Mini tab (Eyi Ndong et al., 2011) puis conserver dans des bocaux contenant du silica gel. Le logiciel BRAHMS (Botanical Research And Herbarium Management System) version 7.6 (www.brahmsonline.com; http://herbaria.plants.ox ac.uk/bol/) a été utilisé la gestion des herbiers et le traitement des données liées à ces échantillons. Les données sur les connaissances ethnobotaniques des Mbènzèlè et des Ngombe ont été collectées en utilisant une enquête semi-structurée qui est l'une des méthodes d'investigation des connaissances endogènes (Zent \& Zent, 2011). L'enquête semi-structurée consiste à interviewer les enquêtés en utilisant un formulaire comprenant des thèmes bien précis à aborder (Codjia \& Yorou, 2014). Les enquêtés ont été interviewés un à un de façon isolée de sorte que les réponses données par un(e) enquêté (e) ne soient pas entendues par celui ou celle qui sera interviewé(e) après.

Identification des spécimens : Les échantillons récoltés (212) ont été provisoirement déposés à l'Herbier de I'ONG Initiative des Champignons et des Plantes du Congo (ICPC). Des doubles ont aussi été déposés au Jardin Botanique National Meise, Belgique (BR). Les observation microscopiques ont été faites d'après (Ndolo Ebika, 2014; 2015). La combinaison des caractères macro- et micromorphologiques a permis d'identifier les spécimens principalement à partir des différentes Flores de champignons d'Afrique publiées entre 1935 et 1997. Les aspects nomenclaturaux ont été vérifiés dans MycoBank (www.mycobank.org) et dans Index Fungorum (www.mycology.net). 
Ebika et al, J. Appl. Biosci. 2018 Les champignons sauvages comestibles et connaissances endogènes des peuples autochtones Mbènzèlè et Ngombe de la République du Congo.

\section{RÉSULTATS}

Nombre d'espèces de champignons utilisées et leur mode de vie : Au total 51 espèces ont été enregistrées comme comestibles dans le milieu d'étude lors des récoltes opportunistes dont 32 ont été citées au cours des enquêtes. Les genres les plus représentées sont Cantharellus, Marasmius, Russula et Termitomyces avec $10,6,5$ et 4 espèces respectivement. Parmi ces 51 espèces, 28 d'entre elles soit $54,9 \%$ sont saprotrophes (poussant sur des bois mort, débris végétaux, terre.) tandis que 23 espèces soit $45,1 \%$ sont symbiotiques (poussant en étroite association avec certains arbres et des termitières).

Correspondance entre les noms scientifiques et les noms locaux (Mbènzèlè et Ngombe) des champignons comestibles : Le tableau 1 fait donne la liste des espèces comestibles enregistrées dans la zone d'étude et fait une comparaison entre les noms locaux Mbènzèlè et les noms Ngombe. Le type de forêt dans lequel les spécimens de ces espèces ont été récoltés ainsi que le substrat sur lequel ces champignons poussaient y sont aussi mentionnés. 
Ebika et al, J. Appl. Biosci. 2018 Les champignons sauvages comestibles et connaissances endogènes des peuples autochtones Mbènzèlè et Ngombe de la République du Congo.

Tableau 1: Correspondance entre les noms scientifiques et les noms locaux (Mbènzèlè et Ngombe) des champignons comestibles dans la zone d'étude.

\begin{tabular}{|c|c|c|c|c|c|}
\hline Familles \& Espèces & No d'herbier & Nom Mbènzèlè & Nom Ngombe & Habitat & Substrat \\
\hline \multicolumn{6}{|l|}{ AMANITACEAE } \\
\hline Amanita loosii Beeli & NEST $712,789,1124,1610$ & Ngombo & Sokeke & FGD & Terre proche d'une termitière \\
\hline \multicolumn{6}{|l|}{ AURICULARIACEAE } \\
\hline Auricularia cornea Ehnerb. & $\begin{array}{l}\text { NEST 1238, 1257, } 1337, \\
1572,1675,2068\end{array}$ & $\begin{array}{l}\text { Makòdou, } \\
\text { Maloyi ma } \\
\text { Sumbu }\end{array}$ & & FMTF, FGD & Bois mort \\
\hline Auricularia delicata (Fr.) Henn. & NEST 746, 1500, 2078, 2112 & $\begin{array}{l}\text { Bòkòdou, } \\
\text { Makòdou. }\end{array}$ & Djè bolo & FMTF & Bois mort \\
\hline \multicolumn{6}{|l|}{ CANTHARELLACEAE } \\
\hline $\begin{array}{l}\text { Cantharellus cibarioides (Heinem.) } \\
\text { Buyck }\end{array}$ & NEST 1367, 1641 & Bilemi ba babika & Bakika, Bika & FGD & Terre \\
\hline Cantharellus congolensis Beeli & $\begin{array}{l}\text { NEST } 852,1166,1593,1773 \text {, } \\
2051,2369\end{array}$ & $\begin{array}{l}\text { Makundu ma } \\
\text { (ba) Bemba. }\end{array}$ & Mbu Bemba & FGD & Terre \\
\hline $\begin{array}{l}\text { Cantharellus cyanoxanthus R. } \\
\text { Heim ex Heinem. }\end{array}$ & NEST 2280, 2333, 2353 & Magnagoua & & FGD & Terre \\
\hline $\begin{array}{l}\text { Cantharellus goossensiæ (Beeli) } \\
\text { Heinem. }\end{array}$ & $\begin{array}{l}\text { NEST 1097, } 1215,1410, \\
1550,1722\end{array}$ & Mokili & Mokili & FGD & Terre \\
\hline Cantharellus longisporus Heinem. & AGB 26; NEST 737, 744 & $\begin{array}{l}\text { Membouambou } \\
\text { a }\end{array}$ & & FGD & Terre \\
\hline Cantharellus miniatescens Heinem. & NEST 697, 1164, 1256, 1968 & Mokili & & FGD & Terre \\
\hline Cantharellus rhodophyllus Heinem. & NEST 856 & Misengui & $\begin{array}{l}\text { Mokili, Niè } \\
\text { mangouongouo }\end{array}$ & FGD & Terre \\
\hline $\begin{array}{l}\text { Cantharellus rufopunctatus var. } \\
\text { rufopunctatus (Beeli) Heinem }\end{array}$ & NEST 708, 1224, 1239, 1493 & & Mangouongouo & FGD & Terre \\
\hline Cantharellus sp.2 & NEST 780 & Mangouongouo & $\begin{array}{l}\text { Madzagoua, } \\
\text { Mondzoumbou }\end{array}$ & FGD & Terre \\
\hline $\begin{array}{l}\text { Cantharellus subincarnatus } \\
\text { Eyssartier \& Buyck }\end{array}$ & $\begin{array}{l}\text { AGB 28; NEST 1828, 2175, } \\
2297\end{array}$ & $\begin{array}{l}\text { Molapa, } \\
\text { Mossengi }\end{array}$ & & FGD & Terre \\
\hline $\begin{array}{l}\text { Craterellus aureus Berk. \& M.A. } \\
\text { Curtis. }\end{array}$ & $\begin{array}{l}\text { NEST } 787,1364,1398,1540 \text {, } \\
1766\end{array}$ & $\begin{array}{l}\text { (Ba)Bika, Belemi } \\
\text { ba babika }\end{array}$ & (Ba)Bika, Kaasa & FGD & Terre \\
\hline \multicolumn{6}{|l|}{ COPRINACEAE } \\
\hline Coprinus lagopus (Fr.) Fr. & NEST 2320 & Tokamòkòlò & & $\mathrm{BR}$ & Régime mort de palmier. \\
\hline \multicolumn{6}{|l|}{ CORTINARIACEAE } \\
\hline Gymnopilus zenkeri (Henn.) Singer & $\begin{array}{l}\text { AGB 19; NEST 756, 806, } \\
\text { 1392, 1843, 2143, 2179, } \\
2209,2327\end{array}$ & $\begin{array}{l}\text { Mabòbòlò, } \\
\text { Mayita, } \\
\text { Moudoutou }\end{array}$ & & $\begin{array}{l}\text { BR, FGD, } \\
\text { FMTF, Sc }\end{array}$ & Bois mort, Sciures des grumes \\
\hline LEPIOTACEAE & & & & & \\
\hline
\end{tabular}


Ebika et al, J. Appl. Biosci. 2018 Les champignons sauvages comestibles et connaissances endogènes des peuples autochtones Mbènzèlè et Ngombe de la République du Congo.

\begin{tabular}{|c|c|c|c|c|c|}
\hline $\begin{array}{l}\text { Macrolepiota africana (Heim) } \\
\text { Heinem. }\end{array}$ & $\begin{array}{l}\text { NEST } 1254,1274,1376, \\
1400,1634,1857\end{array}$ & Gombanda & $\begin{array}{l}\text { Bòngò kema, } \\
\text { Sàkà kótó }\end{array}$ & CM, FMTF & Terre \\
\hline $\begin{array}{l}\text { Macrolepiota dolichaula (Berk. \& } \\
\text { Broome) Pegler \& R.W. Rayner }\end{array}$ & $\begin{array}{l}\text { NEST } 1235,1372,1382, \\
1635,1739,1895\end{array}$ & Gombanda & Sàkà kótó & FMTF & Terre \\
\hline \multicolumn{6}{|l|}{ LYOPHYLLACEAE } \\
\hline $\begin{array}{l}\text { Termitomyces cf. entolomoides } \\
\text { Heim }\end{array}$ & $\begin{array}{l}\text { NEST 1151, 2148, 2194, } \\
\text { 2223, } 2306\end{array}$ & Dikaliké & & FMTF, FGD & Termitière souterraine \\
\hline $\begin{array}{l}\text { Termitomyces microcarpus (Berk. \& } \\
\text { Br.) Heim }\end{array}$ & NEST 1160, 1290, 1354 & Ndzokobouka & $\begin{array}{l}\text { Mobóli, } \\
\text { Ndòbòlòbò, } \\
\text { Ndzokobouka }\end{array}$ & FMTF & $\begin{array}{l}\text { Pâte jaunâtre sur débris végétaux, bois mort, } \\
\text { par terre ou sur de termitière }\end{array}$ \\
\hline $\begin{array}{l}\text { Termitomyces robustus (Beeli) } \\
\text { Heim }\end{array}$ & NEST 1369 & Kouakoualakoua & Motikalike & FMTF & Termitière souterraine \\
\hline Termitomyces striatus (Beeli) Heim & $\begin{array}{l}\text { NEST } 1122,1269,1280 \\
1289,1291,1340,1344 \\
1352,1893,1916\end{array}$ & Mosèlèlè & Moboli, Mosèlèlè & FMTF, FGD & Termitière souterraine \\
\hline \multicolumn{6}{|l|}{ MARASMIACEAE } \\
\hline $\begin{array}{l}\text { Gerronema holochlorum (Berk. \& } \\
\text { Br.) Pegler }\end{array}$ & NEST 2003, 2016, 2035 & Malikouòlòlò & & FGD & Bois mort \\
\hline Marasmius bekolacongoli Beeli & NEST 1942, 2007, 2245 & (Ma)Sèkèsèkè & & FMTF, FGD & Bois mort, débris végétaux \\
\hline Marasmius buzungolo Singer & $\begin{array}{l}\text { NEST } 727,833,1120,1576, \\
1589,1643,1644,1761,2288\end{array}$ & Ndjingo & $\begin{array}{l}\text { Mondjingola } \\
\text { ndjingola; } \\
\text { Ndjingola }\end{array}$ & FMTF & Débris végétaux, terre \\
\hline Marasmius haediniformis Singer & NEST 2206, 2250, 2287 & Masèkèsèkè & & FMTF & Bois mort, débris végétaux \\
\hline Marasmius sierraleonis Beeli & NEST 1110, 1663, 2196 & Masèkèsèkè & & FMTF & Débris végétaux, feuilles mortes \\
\hline Marasmius sp.1 & $\begin{array}{l}\text { NEST 1198, 1551, 1904, } \\
1972,2149,2150\end{array}$ & Gbasèlè & & FMTF, FGD & $\begin{array}{l}\text { Toujours sur rhizome, jeune pousse, tige } \\
\text { matûre, gaine foliaire de Haumania } \\
\text { danckelmaniana ou vivant }\end{array}$ \\
\hline $\begin{array}{l}\text { Marasmius staudtii Henn. var. } \\
\text { staudtii }\end{array}$ & NEST $865,1244,1666$ & & Toulou Gbasèlè & FMTF & Débris végétaux \\
\hline Trogia infundibuliformis Berk. \& Br & $\begin{array}{l}\text { NEST } 864,1204,1208,1332, \\
1355,1510,1620,1808\end{array}$ & Kingili & & FMTF, FGD & Bois mort \\
\hline \multicolumn{6}{|l|}{ MYCENACEAE } \\
\hline Mycena myxocaulis Pegler & NEST1927, 2165, 2266 & Mbaso & & FMTF & Bois mort \\
\hline \multicolumn{6}{|l|}{ PHYSALACRIACEAE } \\
\hline Armillaria heimii Pegler & $\begin{array}{l}\text { NEST } 729,1085,1145,1161, \\
1177,1192,1440,1955\end{array}$ & & Mawòlouwòlou & FMTF, FGD & Bois mort \\
\hline
\end{tabular}


Ebika et al, J. Appl. Biosci. 2018 Les champignons sauvages comestibles et connaissances endogènes des peuples autochtones Mbènzèlè et Ngombe de la République du Congo.

\begin{tabular}{|c|c|c|c|c|c|}
\hline Oudemansiella sp. & NEST 2325, 2336, 2341 & & Mawòlouwòlou & FGD & Bois mort \\
\hline \multicolumn{6}{|l|}{ PLEUROTACEAE } \\
\hline $\begin{array}{l}\text { Pleurotus tuber-regium (Rumph. ex } \\
\text { Fr.) Singer }\end{array}$ & $\begin{array}{l}\text { NEST } 758,1255,1271,1378, \\
1516\end{array}$ & $\begin{array}{l}\text { Gombo; Toulou } \\
\text { yaka }\end{array}$ & Kououtou & FMTF, FGD & Sur une sorte de boule appelée Sclérote. \\
\hline \multicolumn{6}{|l|}{ PLUTEACEAE } \\
\hline Pluteus sp.1 & NEST 2207 & Mbèlèkoumou. & & FMTF & Bois mort \\
\hline Volvariella volvacea (Bull.) Singer & AGB 20 & Mambèlèkouma & & Scierie & Mélange de terre plus débris de bois mort \\
\hline \multicolumn{6}{|l|}{ POLYPORACEAE } \\
\hline $\begin{array}{l}\text { Echinochaete brachypora (Mont.) } \\
\text { Ryv. }\end{array}$ & $\begin{array}{l}\text { NEST } 703,721,1188,1275, \\
1567,1812,2177\end{array}$ & $\begin{array}{l}\text { Bèbèsou ba } \\
\text { basoumbou }\end{array}$ & Koto lé Seko & FMTF, FGD & Bois mort \\
\hline Lentinus brunneofloccosus Pegler & NEST 1279, 1335, 1338 & & & FMTF & Bois mort \\
\hline Lentinus sajor-caju (Fr.) Fr. & $\begin{array}{l}\text { NEST } 705,1091,1284,1430, \\
2231\end{array}$ & $\begin{array}{l}\text { Koombili, } \\
\text { Ndzambe } \\
\text { sounda. }\end{array}$ & $\begin{array}{l}\text { Botótó, Koombili, } \\
\text { Moyakayaka. }\end{array}$ & BR, FMTF & Bois mort \\
\hline Lentinus squarrosulus Mont. & $\begin{array}{l}\text { NEST 750, 781, 1301, 1351, } \\
\text { 1353, 1381, 1577, 1747, } \\
\text { 1897, } 2082\end{array}$ & $\begin{array}{l}\text { Bomòngò, } \\
\text { Bototo, Mboko. }\end{array}$ & $\begin{array}{l}\text { Koombili, Ngebe } \\
\text { aya. }\end{array}$ & $\begin{array}{l}\text { FMTF, FGD, } \\
\text { CBC, CM }\end{array}$ & Bois mort \\
\hline Polyporus tenuiculus (P.Beauv.) Fr. & $\begin{array}{l}\text { NEST } 699,733,757,857 \\
1169,1436,1591,1716 \\
1856,1940,2081,2151\end{array}$ & Bokata & & FMTF, FGD & Bois mort \\
\hline \multicolumn{6}{|l|}{ RUSSULACEAE } \\
\hline $\begin{array}{l}\text { Lactifluus gymnocarpus (R. Heim } \\
\text { ex Singer) Verbeken }\end{array}$ & NEST1103, 1770, 1913 & Ndjoumboulela & Mandòngò & FGD & Terre \\
\hline Lactarius sp.1 & NEST 2265 & Mandòngò & & FGD & Bois mort \\
\hline Russula roseostriata Buyck & $\begin{array}{l}\text { NEST 1168, 1380, } 1558, \\
1573\end{array}$ & $\begin{array}{l}\text { Mombindombind } \\
0\end{array}$ & Mombindombindo & FGD & Feuilles mortes, terre \\
\hline Russula sp.1 & NEST 1098 & Ewayi & Ewayi & FGD & Terre \\
\hline Russula sp.2 & NEST 1115 & & Nie mandòngò & FGD & Terre \\
\hline Russula sp.3 & NEST 1608 & Mandòngò & & FGD & Terre \\
\hline Russula sp.4 & NEST 1538 & & Mandòngò & FGD & Terre \\
\hline \multicolumn{6}{|l|}{ SARCOSCYPHACEAE } \\
\hline Cookeina speciosa (Fr.) Dennis & $\begin{array}{l}\text { NEST } 735,1416,1640,2009, \\
2073,2146\end{array}$ & $\begin{array}{l}\text { Tokomba, } \\
\text { Tokosa }\end{array}$ & Totokomba & FMTF, FGD & Bois mort \\
\hline $\begin{array}{l}\text { Cookeina tricholoma (Mont.) } \\
\text { Kuntze }\end{array}$ & NEST 736, 2071, 2072 & Tokomba. & & FMTF & Bois mort \\
\hline $\begin{array}{l}\text { Collybia confluens (Pers. ex Fr.) } \\
\text { Kummer }\end{array}$ & NEST 1415, 1884 & & & BV & Débris végétaux \\
\hline
\end{tabular}


Usages des champignons : A la différence des plantes qui sont utilisées comme des aliments, des médicaments, des produits de beauté et aussi dans des pratiques magiques par les peuples autochtones de la zone d'étude (Bahuchet, 1991; Lewis, 2002), les champignons sont principalement classés en deux groupes: comestibles ou non comestibles. Ils sont principalement récoltés pour l'autoconsommation. Seuls trois espèces sont utilisées dans la pharmacopée traditionnelle. Il s'agit de Auricularia cornea et $A$. delicata. Ces dernières sont bouillies et la solution résultante administrée par voie orale permet de traiter la diarrhée. En outre, Lentinus squarrosulus est utilisée comme purge après préparation. Sept espèces notamment Cantharellus congolensis, $C$. cyanoxanthus, Lactifluus gymnocarpus, Lentinus sajor-caju, $L$. squarrosulus, Marasmius buzungolo et Russula roseostriata font l'objet d'une vente de porte-à-porte ou encore d'échange sous forme de Troc. Ce troc est uniquement fondé sur l'échange de ces champignons avec un bol de farine de manioc ou encore avec du sel de cuisine.

Composition, signification et variation de la nomenclature locale des CSC : Les noms vernaculaires sont attribués aux champignons soit par rapport à la ressemblance du champignon avec un autre être vivant ou une partie d'un être vivant, soit par rapport à leur écologie ou encore par rapport à la consistance que donne le champignon à la soupe. Le Tableau 2 fournit les détails sur la signification des noms vernaculaires de certains champignons. 
Ebika et al, J. Appl. Biosci. 2018 Les champignons sauvages comestibles et connaissances endogènes des peuples autochtones Mbènzèlè et Ngombe de la République du Congo.

Tableau 2 : Signification de quelques noms vernaculaires des espèces fongiques de la zone d'étude

\begin{tabular}{|c|c|c|c|}
\hline Nom scientifique & Nom Mbènzèlè/ signification & Nom Ngombe/ signification & Caractère utilisé \\
\hline Armillaria heimii & & $\begin{array}{l}\text { Mawòlouwòlou/ nom donné au champignon } \\
\text { à cause de l'aspect gluant de la sauce. }\end{array}$ & $\begin{array}{l}\text { Consistance de la sauce après cuisson } \\
\text { du champignon }\end{array}$ \\
\hline Auricularia cornea & Maloyi ma Sumbu/ Oreilles du chimpanzé & & $\begin{array}{l}\text { Ressemblance avec une partie d'un } \\
\text { animal }\end{array}$ \\
\hline Auricularia delicata & & Djè bolo/ Oreille du chien & $\begin{array}{l}\text { Ressemblance avec une partie d'un } \\
\text { animal }\end{array}$ \\
\hline Cantharellus cibarioides & Bilemi ba babika/ Langues des Perroquets & (Ba)Bikal Perroquet(s) & $\begin{array}{l}\text { Ressemblance avec une partie d'un } \\
\text { animal ou à l'animal entier }\end{array}$ \\
\hline C. congolensis & $\begin{array}{l}\text { Makundu ma (ba)Bembal Boyaux du Cephalophus } \\
\text { sylvicultor }\end{array}$ & $\begin{array}{l}\text { Mbu Bembal Boyaux du Cephalophus } \\
\text { sylvicultor }\end{array}$ & $\begin{array}{l}\text { Ressemblance avec une partie d'un } \\
\text { animal }\end{array}$ \\
\hline Craterellus aureus & $\begin{array}{l}\text { (Ba)Bika, Belemi ba babika/ Perroquet(s), langues } \\
\text { des Perroquets }\end{array}$ & (Ba)Bika/ Perroquet(s) & $\begin{array}{l}\text { Ressemblance avec une partie d'un } \\
\text { animal ou à l'animal entier }\end{array}$ \\
\hline Coprinus lagopus & Tokamòkòlò/champignon apparaissant le soir & & Phénologie de l'espèce \\
\hline Echinochaete brachypora & Bèbèsou ba basoumbou/ Lèvres des chimpanzés & $\begin{array}{l}\text { Koto lé Seko/ lèvres vaginales } d u \\
\text { chimpanzé }\end{array}$ & $\begin{array}{l}\text { Ressemblance avec une partie d'un } \\
\text { animal }\end{array}$ \\
\hline Marasmius sp.1 & $\begin{array}{l}\text { Gbasèlè/ nom vernaculaire de la plante herbacée } \\
\text { (Haumania danckelmaniana, Marantaceae) sur } \\
\text { laquelle pousse ce champignon. }\end{array}$ & & $\begin{array}{l}\text { Etroite relation entre le champignon et } \\
\text { son support }\end{array}$ \\
\hline Mycena myxocaulis & $\begin{array}{l}\text { Mbaso/ nom vernaculaire du bois (Dialium spp., } \\
\text { Leguminosae-Caesalpinioideae) sur lequel pousse } \\
\text { ce champignon. }\end{array}$ & & $\begin{array}{l}\text { Etroite relation entre le champignon et } \\
\text { son support }\end{array}$ \\
\hline Oudemansiella sp. & $\begin{array}{l}\text { Mawòlouwòlou/ nom donné au champignon à cause } \\
\text { de l'aspect gluant de la sauce. }\end{array}$ & & $\begin{array}{l}\text { Consistance de la sauce après cuisson } \\
\text { du champignon }\end{array}$ \\
\hline $\begin{array}{l}\text { Termitomyces cf. } \\
\text { entolomoides }\end{array}$ & $\begin{array}{l}\text { Dikaliké/ Orphelin, Solitaire car ce champignon } \\
\text { pousse toujours de façon solitaire }\end{array}$ & & Phénologie de l'espèce \\
\hline T. robustus & & $\begin{array}{l}\text { Motikalike/ Orphelin, Solitaire car ce } \\
\text { champignon pousse toujours de façon } \\
\text { solitaire }\end{array}$ & Phénologie de l'espèce \\
\hline T. striatus & Mosèlèlè/ nom vernaculaire des termites & Mosèlèlè/ nom vernaculaire des termites & $\begin{array}{l}\text { Correspondance avec les insectes } \\
\text { partageant le même milieu }\end{array}$ \\
\hline
\end{tabular}




\section{DISCUSSION}

Le présent travail a montré que les genres Cantharellus, Marasmius, Russula et Termitomyces sont les plus représentées avec dix (10), six (06), cinq (05) et quatre (04) espèces respectivement. En RDC, Madamo et al. (2017) reportent pour la Région de Kikwit que ce sont les genres Marasmius, Termitomyces, Cantharellus, Lentinus et Auricularia qui possèdent plus d'espèces comestibles. Chez les peuples autochtones Bofi de la Lobaye en RCA, Termitomyces est le genre le plus riche en espèces comestibles (Malaisse et al., 2008). Au total 51 espèces de champignons comestibles ont été répertoriées dans la partie Nord du Congo dont sept (07) notamment Cantharellus goossensiæ, Echinochaete brachypora, Marasmius haediniformis, M. sierraleonis, M. staudtii var. staudtii, Mycena myxocaulis et Polyporus tenuiculus sont reportées pour la première fois comme comestibles en

\section{CONCLUSION}

L'état des connaissances scientifiques sur les champignons sauvages comestibles (CSC) en République du Congo reste encore embryonnaire. Au Nord du Congo en général et dans les villages Bomassa, Makao et Thanry en particulier, les connaissances sur les CSC sont détenues par deux groupes de peuples autochtones: les Ngombe (ou Aka) vivant à Bomassa et les Mbènzèlè (ou Baka) vivant à Makao et Thanry et aussi à Bomassa. Les résultats de cette étude ne représentent qu'environ $50 \%$ de la connaissance endogène de ces deux groupes ethniques étudiés. Ainsi, face à la destruction des forêts et aux changements

\section{RÉFÉRENCES BIBLIOGRAPHIQUES}

Bahuchet S, 1991. Les Pygmées d'aujourd'hui en Afrique centrale. Journal des Africanistes, Société des Africanistes, 61(1): 5-35.

Bahuchet S, et Thomas JMC, 1986. Linguistique et histoire des Pygmées de l'Ouest du Bassin Congolais. Sprache und Geschichte in Afrika, 7(2): 73-103.

Boa E, 2006. Produits Forestiers Non Ligneux 17. Champignons comestibles sauvages. FAO, Rome. $157 \mathrm{p}$.

Bouka Owoko R, et N'zobo RE, 2011. Les peuples autochtones de la République du Congo: discrimination et esclavage. OCDH, Brazzaville, $68 \mathrm{p}$.

Buyck B, 1994. UBWOBA: Les champignons comestibles de l'Ouest du Burundi. Administration Générale
Afrique. Ce nombre total d'espèces est considérablement élevé par rapport aux neuf (09) espèces reportées pour toute la République du Congo dans les travaux antérieurs (Heim, 1977; Rammeloo \& Walleyn, 1993). Cette augmentation en nombre correspond bien à l'hypothèse émise par Boa (2006) qui stipulait déjà qu'il existerait un usage large des champignons comestibles au Congo. $\mathrm{Ce}$ nombre (51) bien qu'étant encore partiel pour tout le Congo est nettement inférieur aux 74 espèces enregistrées en République Démocratique du Congo (Heim, 1977; Rammeloo \& Walleyn, 1993; Dibaluka et al., 2010), aux 60 espèces du Bénin (Yorou et al., 2017) et largement supérieur aux 25 espèces du Burundi (Rammeloo \& Walleyn, 1993; Buyck, 1994) et aux 24 espèces de la Rép. Centrafricaine (Heim, 1977; Rammeloo \& Walleyn, 1993; Malaisse et al., 2008).

d'habitudes de ces groupes par rapport à l'influence de la société moderne sur leur culture, d'intenses missions de terrain auprès de ces populations sont donc très nécessaires afin de conserver ces connaissances traditionnelles sur les CSC. Aussi, ces études devraient être étendues à d'autres parties du Pays pour permettre d'avoir un guide sur les Champignons Sauvages Comestibles en République du Congo. La réalisation d'un tel ouvrage est l'une des priorités de recherche que proposait Boa (2006) pour la meilleure connaissance de la mycoflore africaine.

de la Coopération au Développement Bruxelles. $123 \mathrm{p}$.

Codjia JEI, et Yorou NS, 2014. Ethnicity and gender variability in the diversity of Wild Useful Fungi in Pobe region, Benin. Journal of Applied Biosciences, 78: $6729-6742$.

De Kesel A, 2001. A mushroom dryer for the travelling mycologist. Field Mycology, 2(4): 131-133.

Dibaluka MS, Lukoki LF, De Kesel A, et Degreef J, 2010. Essais de culture de quelques champignons lignicoles comestibles de la région de Kinshasa (R.D. Congo) sur divers substrats lignocellulosiques. Biotechnol. Agron. Soc. Environ., 14(3): 417-422.

Eyi Ndong H, Degreef J, et De Kesel A, 2011. Champignons comestibles des forêts denses 
d'Afrique centrale: Taxonomie et identification. $\mathrm{ABC}$ taxa, 10, Meise. $254 \mathrm{p}$.

Gillet J-F, et Doucet J-L, 2012. A commented checklist of woody plants in the Northern Republic of Congo. Plant Ecology and Evolution, 145(2): 258-271.

Harris DJ, 2002. The vascular plants of the DzangaSangha reserve. National Botanic Garden (Belgium), Meise. 274 p.

Heim R, 1977. Termites et champignons: les champignons termitophiles d'Afrique Noire et d'Asie Méridionale. Boubée, Paris. 205 p.

Kimpouni V, Lejoly J, et Lisowski S, 1992. Les Eriocaulaceae du Congo. Fragmenta Floristica et Geobotanica, 37(1): 127-145.

Lewis J, 2002. Forest hunter-gatherers and their world: a study of the Mbendjele Yaka Pygmies of CongoBrazzaville and their secular and religious activities and representations. London School of Economics and Political Science, London, 311 p.

Madamo MF, Lubini A, Lukoki F, et Kidikwadi E, 2017. Champignons comestibles de la région de Kikwit en République Démocratique du Congo : Approche écologique, nutritionnelle et socioéconomique. International Journal of Innovation and Applied Studies, 21(1): 124-136.

Malaisse F, De Kesel A, et Lognay G, 2008. Diversité des champignons consommés par les pygmées Bofi de la Lobaye (République centrafricaine). GeoEco-Trop, 32: 1-8.

Moutsamboté J-M, Yumoto T, Mitani M, Nishihara T, Suzuki S, et Kuroda S, 1994. Vegetation and list of plant species identified in the Nouabalé-Ndoki Forest, Congo. Tropics, 3(3/4): 277-293.

Ndolo Ebika ST, 2014. Rapport de stage sur les techniques d'identification des champignons au Jardin Botanique National de Belgique (16 Septembre - 1 Octobre 2013). Initiative des Champignons et des Plantes du Congo (ICPC), Brazzaville, $35 \mathrm{p}$.

Ndolo Ebika ST, 2015. Rapport de stage sur la microscopie des macromycètes du Congo. ICPC \& LEB, Brazzaville, Parakou, 36 p.

Ndolo Ebika ST, Degreef J, Harris DJ, Morgan D, De Kesel A, et Sanz C. (2013a). Safeguarding traditional knowledge of wild edible plants and mushrooms in Bayaka communities (Republic of Congo). Poster., International conference in Nutrition and Food Production in the Congo Basin. Brussels, Belgium: http://kaowarsom.be /documents/Conference_Posters/Ebika.pdf.

Ndolo Ebika ST, Harris DJ, et Degreef J. (2013b). Safeguarding traditional knowledge of wild edible plants and mushrooms in Bayaka communities (Republic of Congo). In J. Rammeloo (Ed.), International Conference on Nutrition and Food Production in the Congo Basin (Vol. 17, pp. 107-108). Brussels: Royal Academy for Overseas Sciences: National Committee for Biological Sciences.

Olivero J, Fa JE, Farfán MA, Lewis J, Hewlett B, Breuer $\mathrm{T}$, Carpaneto $\mathrm{GM}$, Fernández $\mathrm{M}$, Germi $\mathrm{F}$, Hattori S, Head J, Ichikawa M, Kitanaishi K, Knights J, Matsuura N, Migliano A, Nese B, Noss A, Ongbwa Ekoumou D, Paulin P, Real R, Riddell M, Stevenson EGJ, Toda M, Vargas JM, Yasuoka $H$, et Nasi $R, 2016$. Distribution and numbers of pygmies in Central African forests. PLoS ONE, 11(1): e0144499.

Rammeloo J, et Walleyn R, 1993. The edible fungi of Africa south of the Sahara: a literature survey. National Botanic Garden of Belgium, Meise. 62 p.

Seshadri RJ, 2005. Pygmies in the Congo Basin and Conflict. ICE Case Studies, 163.

Vennetier P, 1966. Géographie du Congo-Brazzaville. Gauthier-Villars, Paris. 169 p.

Yorou NS, Codjia JEI, Sanon E, et Tchan KI, 2017. Les champignons sauvages utiles: une mine d'or au sein des forêts béninoises Bulletin de la Recherche Agronomique du Bénin, EAFFC: 31 45.

Zent S, et Zent EL. (2011). Ethnobiological methods for ethnomycological research: quantitative approaches. In A. B. Cunningham \& X. Yang (Eds.), Mushrooms in forests and woodlands: resource management, values and local livelihoods (pp. 61-85). London: Eartscan. 